\title{
High Throughput Screening for Mammography using a Human- Computer Interface with Rapid Serial Visual Presentation (RSVP)
}

\author{
Chris Hope ${ }^{\mathrm{a}}$, Annette Sterr ${ }^{\mathrm{a}}$, Premkumar Elangovan ${ }^{\mathrm{b}}$, Nicholas Geades $^{\mathrm{b}}$, \\ David Windridge ${ }^{\mathrm{b}}$, Ken Young ${ }^{\mathrm{c}}$, Kevin Wells ${ }^{\mathrm{b}}$ \\ ${ }^{\mathrm{a} C l i n i c a l}$ Neuroscience Research Team, University of Surrey, Guildford, UK, GU2 7XH; \\ ${ }^{\mathrm{b}}$ Centre for Vision, Speech and Signal Processing, University of Surrey, Guildford, UK, GU2 7XH; \\ ${ }^{c}$ National Coordinating Centre for the Physics of Mammography, Royal Surrey County Hospital, Guildford, \\ Surrey. UK
}

\begin{abstract}
The steady rise of the breast cancer screening population, coupled with data expansion produced by new digital screening technologies (tomosynthesis/CT) motivates the development of new, more efficient image screening processes. Rapid Serial Visual Presentation (RSVP) is a new fast-content recognition approach which uses electroencephalography to record brain activity elicited by fast bursts of image data. These brain responses are then subjected to machine classification methods to reveal the expert's 'reflex' response to classify images according to their presence or absence of particular targets. The benefit of this method is that images can be presented at high temporal rates $(\sim 10$ per second), faster than that required for fully conscious detection, facilitating a high throughput of image (screening) material. In the present paper we present the first application of RSVP to medical image data, and demonstrate how cortically coupled computer vision can be successfully applied to breast cancer screening. Whilst prior RSVP work has utilised multichannel approaches, we also present the first RSVP results demonstrating discriminatory response on a single electrode with a ROC area under the curve of $0.62-0.86$ using a simple Fisher discriminator for classification. This increases to $0.75-0.94$ when multiple electrodes are used in combination.
\end{abstract}

Keywords: Electroencephalography (EEG), Brain-Computer Interface (BCI), Single-Trial Analysis, Rapid Serial Visual Presentation (RSVP), Breast Cancer, Mammography.

\section{Introduction}

National breast screening programmes face growing demands from the increasing size of screening populations, the larger data sets produced by the next generation of digital imaging technologies (tomosynthesis and breast CT), and within many Western countries, financial pressures to provide greater throughput at reduced costs. In the absence of substantial increases in funding, there is a need to produce faster methods for screening image data and to reduce the timescales needed to train expert radiological observers. In order to respond to these challenges, this work presents the first attempt to harness brain-computer interface (BCI) techniques involving Rapid Serial Visual Presentation (RSVP) in the medical domain. This approach has proved highly successful in analysing large quantities of image data at high rates of throughput in the military arena ${ }^{1-6}$ with area under the receiver operating characteristics (ROC) curve (AUC) statistics of up to $\sim 0.9$. These techniques take advantage of the advanced visual processing capabilities of the human brain by measuring the expert's semi-conscious response, known as the P300, whilst viewing image stimuli in rapid succession $(\sim 10 \mathrm{~Hz})$, wherein the rate of image presentation is faster than that needed for conscious discrimination. Typically the neural response to 'target' images differs compared to 'non-target' images, allowing the detection of target image presence from EEG data through machine learning methods. BCI techniques using RSVP may therefore offer great potential for high throughput medical screening applications, and might also be developed for rapid feedback during training of expert observers. This work presents the first proof-of-concept study to examine the use of RSVP in a medical domain, and has not been published or presented elsewhere before.

Medical Imaging 2013: Image Perception, Observer Performance, and Technology Assessment, edited by Craig K. Abbey, Claudia R. Mello-Thoms, Proc. of SPIE Vol. 8673, 867303

(C) 2013 SPIE · CCC code: $1605-7422 / 13 / \$ 18 \cdot$ doi: 10.1117/12.2007557

Proc. of SPIE Vol. 8673 867303-1 


\section{Methodology}

In the current study we tested a BCI system with two distinct image datasets: (a) mammogram targets containing a single biopsy-proven lesion or simulated lesion with realistic appearance ${ }^{10}$ against non-targets consisting of lesion-free background mammography segments (Fig.1a), and for comparison (b) a set of green and red stimuli set against a set of random blobs as non-targets (Fig.1b). All mammography screening image data were derived from the Optimam image database ${ }^{9}$.

These two experiments allowed us to contrast BCI performance with different stimulus types. In both experiments a series of images was rapidly presented to the observer in 3-second bursts, consisting of 30 images, each presented for $100 \mathrm{~ms}$. Image data were pre-designated as non-target or target although this information was not presented to participants.

Each experiment was split into two phases. Firstly, a classifier training phase, consisting of 608 image bursts, and a classifier testing phase of a further 608 bursts in which different exemplars of the same target and non-target images were presented to the observer (see Figure 1c ). In each phase half the bursts were randomly comprised of entirely nontargets (i.e. non-target bursts), and the other half contained a single target image at a random position within the burst, located between the $4^{\text {th }}$ to the $27^{\text {th }}$ image (i.e. target bursts). Sixteen unique target images were used in each phase (training/testing) of each experiment. In the mammogram experiment, the target images were all mammogram segments containing a mass in the centre of the image, whereas in the comparative colour experiment red squares were used for training stimuli and green squares for testing. A set of 150 unique non-target images were used in each phase. All images were $5 \mathrm{~cm}^{2}$ in size and presented against a grey global background. EEG data were recorded in DC mode with a sampling rate of $1 \mathrm{kHz}$ from 64 standard electrode sites placed according to the 10-20 system ${ }^{7}$ as shown in Fig 3. Impedances were below $5 \mathrm{k} \Omega$, and a $50 \mathrm{~Hz}$ notch filter applied during recording. Eye movements were monitored with bipolar horizontal and vertical electrooculogram electrodes, and an average reference used for analysis.

First a high cut-off filter of $40 \mathrm{~Hz}$ was applied to the raw EEG to remove muscle artefacts. The filtered data were then segmented into $1100 \mathrm{~ms}$ epochs $(-100 \mathrm{~ms}$ to $+1000 \mathrm{~ms})$ relative to the onset of target/non-target presentation. Non-target segments were taken relative to one image from each non-target burst selected at random, excluding the first and last three images of each burst. All segments were then baseline corrected and any that contained eye or muscle artefacts were removed. ROC curves may then be produced via perturbation of the bias value of the decision hyper-plane in this initial trial within the context of a Fisher classification space.

\section{Results \& Discussion}

Fig. 2 displays the average EEG activity for targets and non-targets at electrodes $\mathrm{C} 1, \mathrm{Cz}, \mathrm{C} 2, \mathrm{P} 1, \mathrm{Pz}, \mathrm{P} 2, \mathrm{O} 1, \mathrm{Oz}$ and $\mathrm{O} 2$ for the two observers in the colour experiment and another four volunteers in the mammogram experiment. The potential generated by target stimuli appears similar to the $\mathrm{P} 300 \mathrm{wave}^{8}$, a large positive deflection largest at central-posterior sites. This potential is absent in non-target trials. The larger difference in amplitude between targets and non-targets for mammogram images compared to colour images could be due to the difference in semantic associations between the content of the two datasets, as the P300 potential has been shown to be sensitive to semantic inference associated with various stimuli ${ }^{8}$. Using Fisher discriminant analysis we obtained ROC AUCs of 0.75 and 0.78 for the two participants tested in the colour experiment. In the mammogram experiment we found ROC AUCs of $0.85,0.80,0.94$ and 0.88 for the four participants tested in the mammogram experiment. 


\section{Conclusions \& Further Work}

The current study demonstrates that BCI technology can successfully detect the presence of well-delineated mass targets against normal mammography architecture with a very high degree of accuracy. These results suggest that BCI technology might eventually be developed to efficiently search through large quantities of mammogram screening data. This also suggests that only a small number of electrodes may be needed, facilitating the use of low cost EEG technology and rapid set-up. Such an approach might also find application in 'tuning' the responses of expert observers during clinical training.

The current pilot study was deliberately confined to well-delineated masses, in order to test for an RSVP response and compare this to a strong colour-based visual response. Although differences in signal potentials between the two datasets are evident, the ROC AUC suggests little difference in performance, suggesting that the classification process has performed robustly with respect to differences in EEG input data. This motivates further work in developing more challenging datasets with a larger cohort of expert volunteers. This trial is currently underway and will be reported on in the final paper. We will also report on analyses in optimising the number of electrodes, and in extending the analysis to include a comparison with using support vector machines for decoding a larger range of input image data.

\section{Acknowledgements}

This work was supported by the Engineering and Physical Sciences Research Council [grant number EP/I000992/1]. This work has not been previously submitted, presented or published elsewhere.

\section{REFERENCES}

[1] Touryan, J., Gibson, L., Horne, J. H., and Weber, P., Frontiers in Psychology, 2, 1-8 (2011).

[2] Parra, L. C., Christoforou, C., Gerson, A. D., Dyrholm, M., Luo, A., Wagner, M., Philiastides, M. G., Sajda, P., IEEE signal processing magazine, 25(1), 107-115 (2008).

[3] Acqualagna, L., and Blankertz, B., $33^{\text {rd }}$ Annual International Conference of the IEEE EMBS, 4560-4563 (2011).

[4] Treder, M. S., Schmidt, N. M., and Blankertz, B., Journal of Neural Engineering, 8, 1-12 (2011).

[5] Bigdeley-Shamlo, N., Vankov, A., Ramierez, R. R., Makeig, S., IEEE Transactions on Neural Systems and Rehabilitation Engineering, 16(5), 432-431 (2008).

[6] Sajda, P., Parra, L. C., Hanna, B., and Chang, S. Proceedings of the IEEE, 98(3), 462-478 (2010).

[7] Jasper, H. H., Electroencephalography and Clinical Neurophysiology, 10, 371-375 (1958).

[8] Kutas, M., McCarthy, G., \& Donchin, E., Science, 197, 792-795 (1977).

[9] Looney, P.T.et al. A national digital mammography image resource. British Journal of Radiology, 29, 19 (2012).

[10] Rashidnasab et al. Realistic simulation of breast mass appearance using random walk. Progress in Biomedical

Optics and Imaging - Proceedings of SPIE Vol., 8313, (2012). 
A

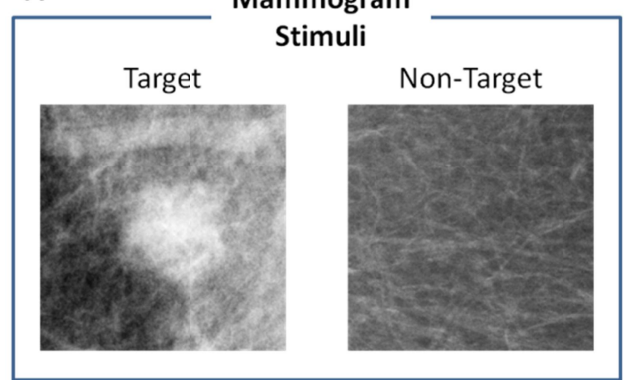

B

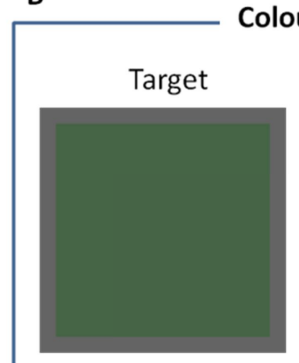

Colour Stimuli

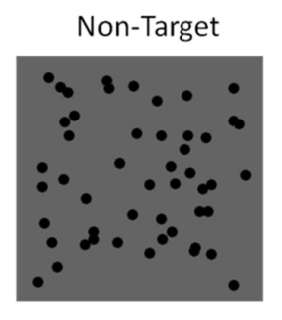

C

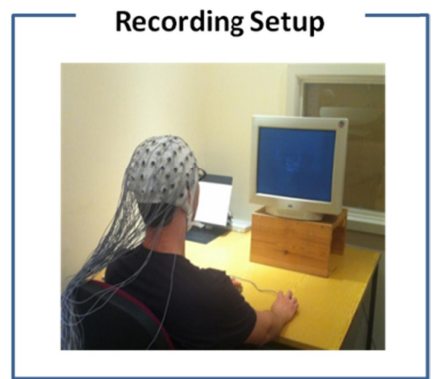

Figure 1. [A] Exemplar target/non-target (lesion/non-lesion) mammographic image segment. [B] Exemplar target/non-target (colour / random blob) colour image segment. [C] The recording set-up used to acquire EEG data whilst an observer undergoes an RSVP trial. The image is presented at the centre of the screen. Images are presented at a small size $\left(5 \mathrm{~cm}^{2}\right)$ in order to minimize eye movements. 

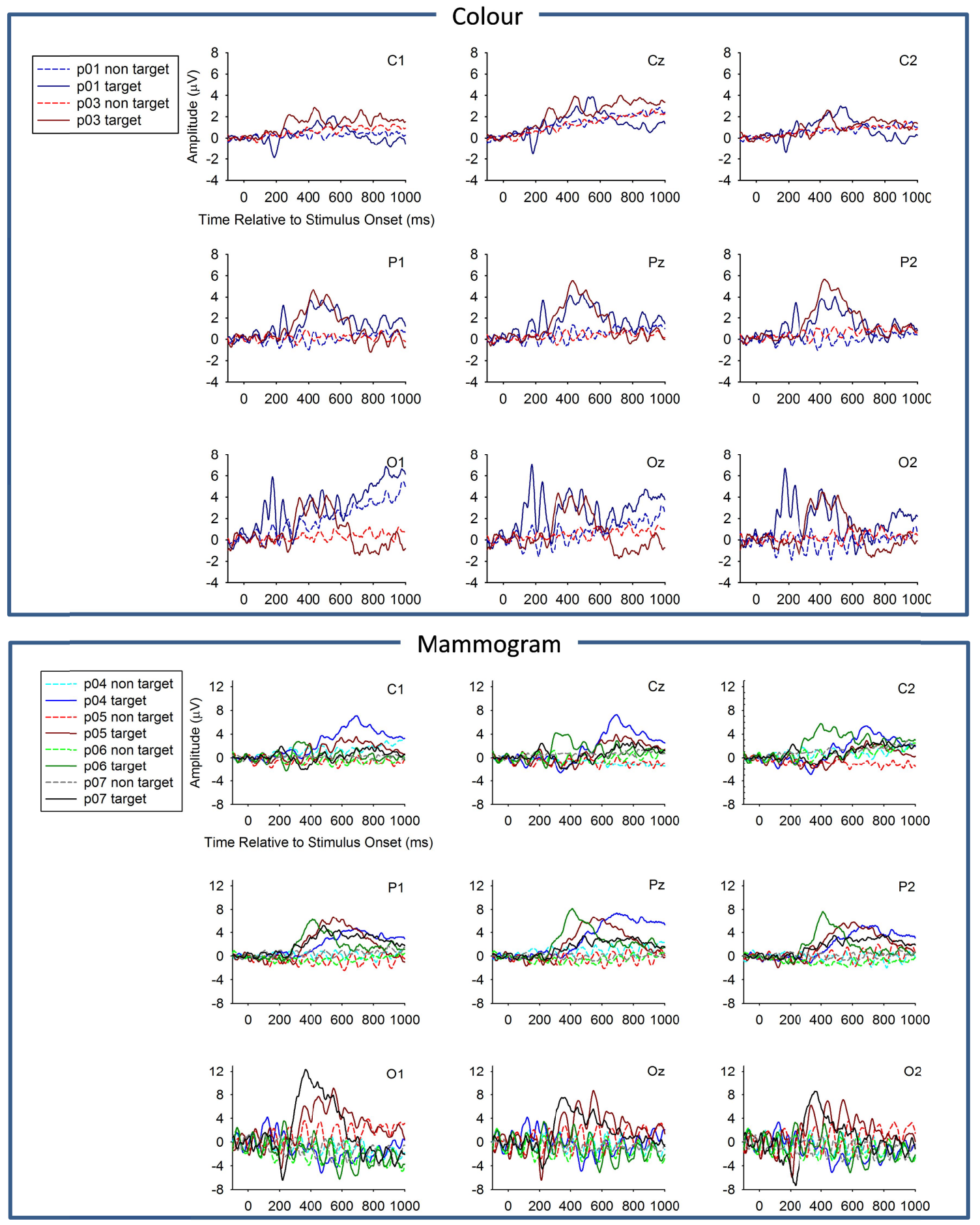

Figure 2. The averaged (mean) EEG activity at electrode sites $\mathrm{C} 1, \mathrm{Cz}, \mathrm{C} 2, \mathrm{P} 1, \mathrm{Pz}, \mathrm{P} 2, \mathrm{O} 1, \mathrm{Oz}$ and $\mathrm{O} 2$ in response to target (solid lines) and non-target stimuli (dashed lines) for and colour stimuli (top) and mammogram stimuli (bottom). Different colours refer to different participants who took part in the study. The standard deviation for each plot was approximately $30 \%$ of the mean amplitude values. 

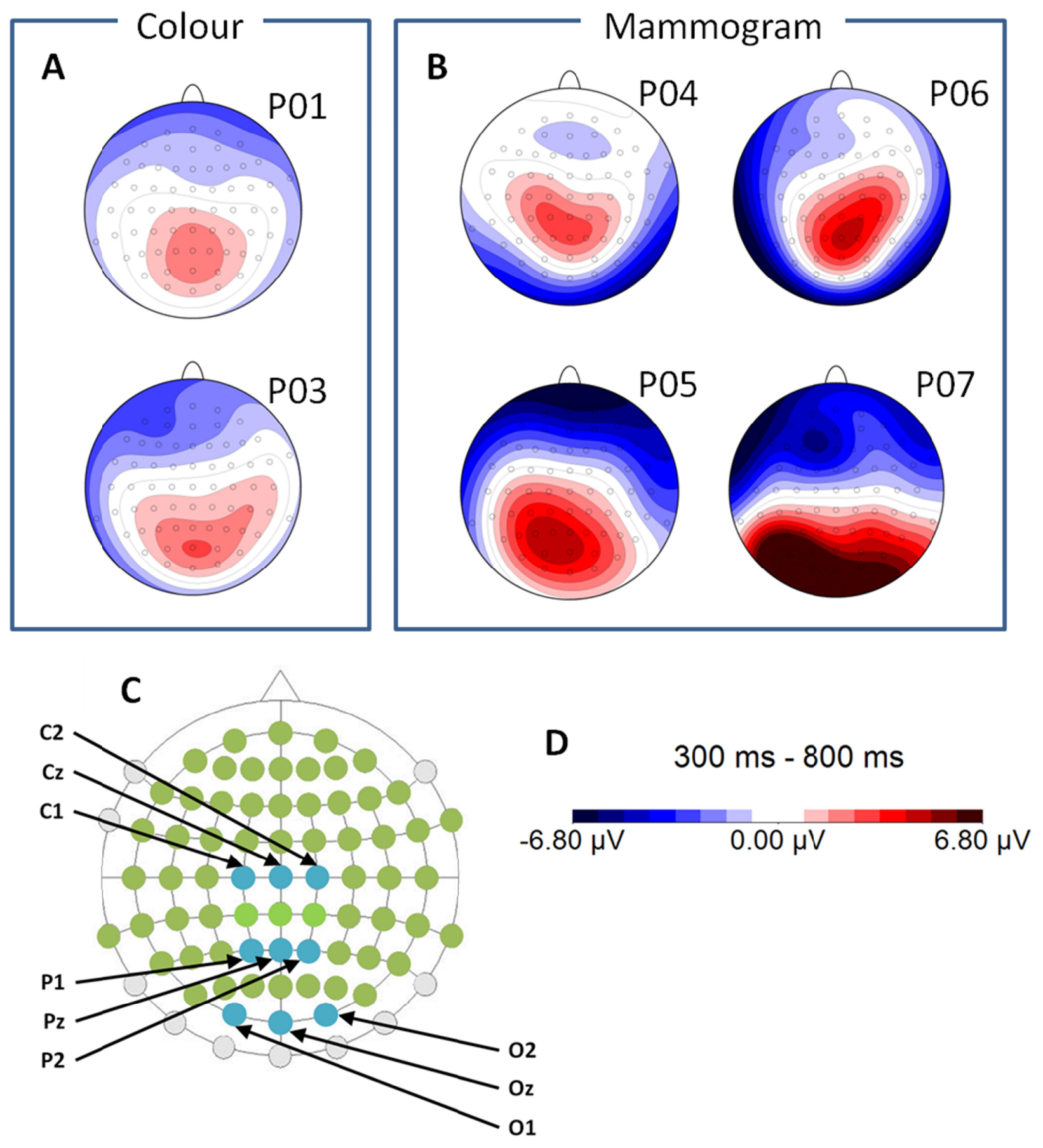

Figure 3. A, B: Difference topographies for the averaged EEG data, calculated by subtracting the activity evoked by target stimuli from the activity in response to non-target stimuli in a time range of $300-800 \mathrm{~ms}$ post stimulus onset. C: Scalp map of standard electrode array placement, the electrodes used to record EEG are highlighted in green. The electrodes where the maximal difference between targets and non-targets was found are highlighted in blue. D: Voltage scale used to plot difference topographies. 


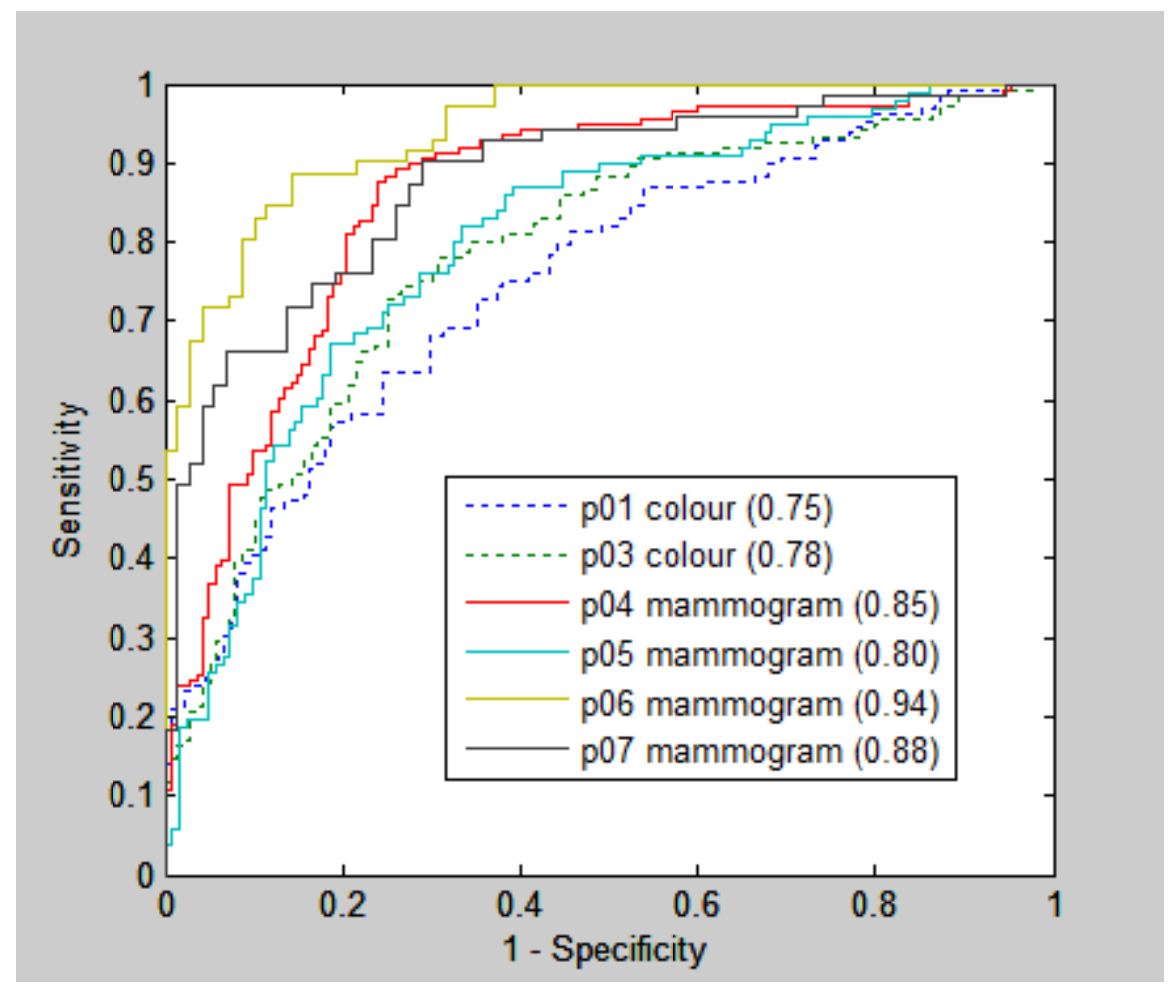

Figure 4. ROC curves from two different volunteers in the colour experiment and four in the mammogram experiment using Fisher Discriminant analysis, AUC values are included in the figure legend.

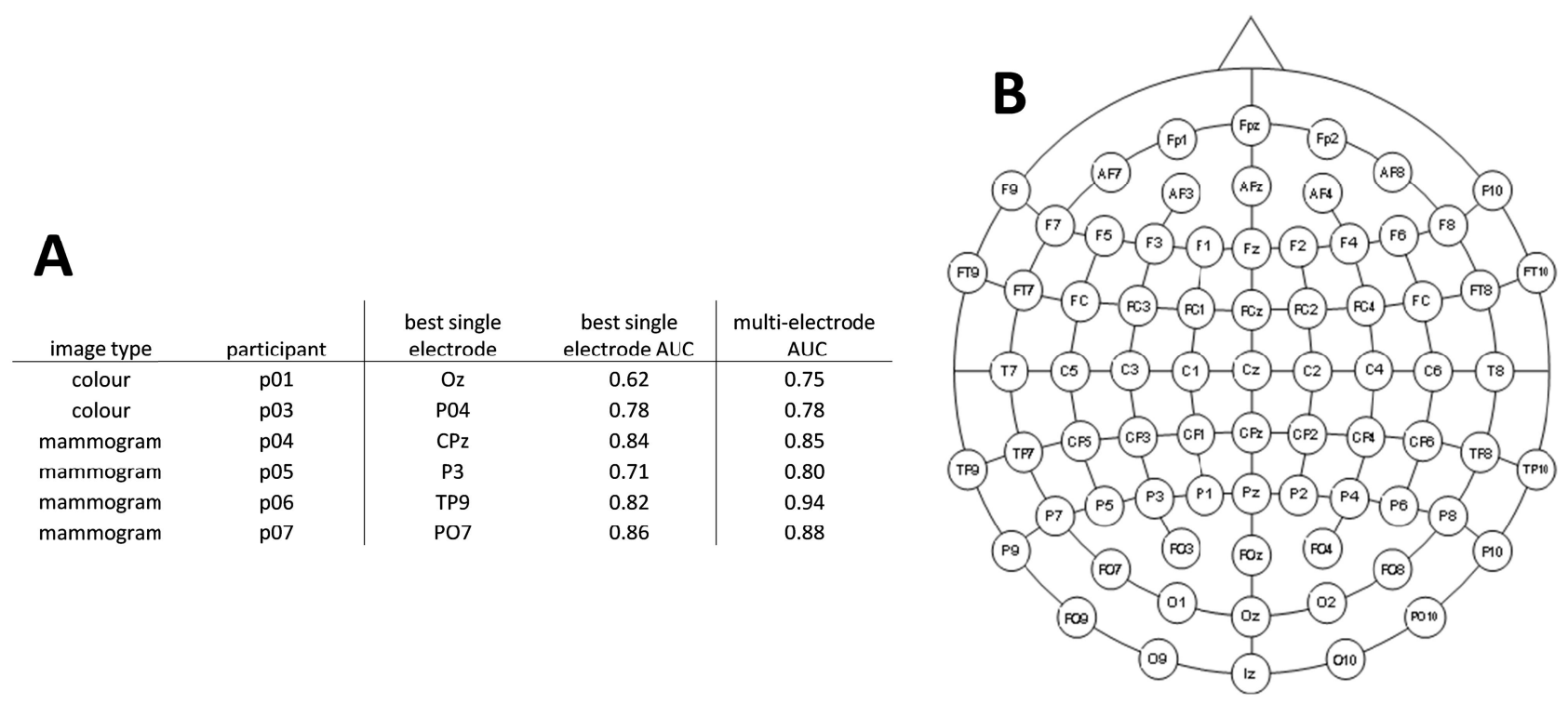

Figure 5. A: The electrode positions across participants of the single best electrode for image classification and their ROC AUC values as well as multi-electrode ROC AUCs for the group of electrodes that produced the best classification performance on the validation data. B: Positions of electrodes on the scalp according to the 10-20 system. 


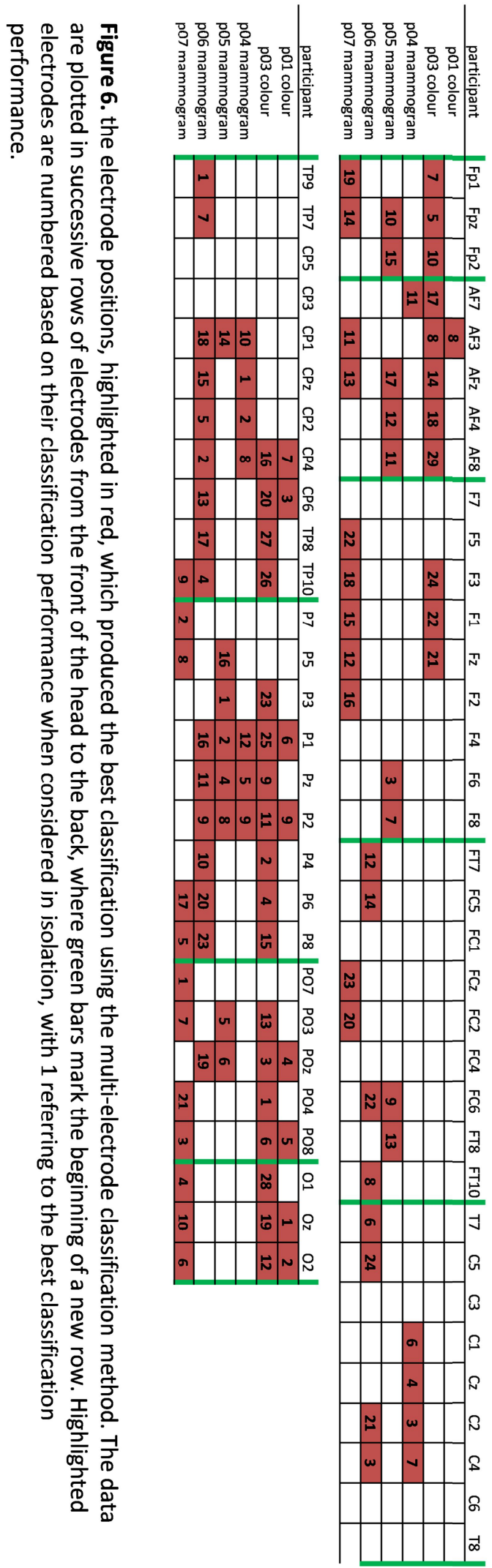

Proc. of SPIE Vol. 8673 867303-8 